\title{
Joint Transceiving Scheme for Multi-beam GEO Satellite Communications System
}

\author{
Yazhe Gao*, Xu Tan**, Xiang Fei**, Bin $\mathrm{Li}^{* *}$ and Jianjun $\mathrm{Wu}^{* *}{ }^{\dagger}$ \\ *ICES, Beijing Information S\&T University, Beijing, P.R.China \\ **Institution of Advanced Communications, Peking University, Beijing, P.R. China \\ E-mail: ${ }^{\dagger}$ just@ pku.edu.cn
}

\begin{abstract}
In multi-beam satellite communication systems, users suffer from 3dB power attenuation in beam-edge area. This problem also exists in terrestrial cellular network. To solve this problem, terrestrial system employs joint transceiving that makes adjacent cells transmit signals together. The purpose of this paper is to propose a method of joint transceiving by compositing beams for satellite systems. The equal-gain combination algorithm is presented to simplify beams from component beams. Then we propose a joint transceiving scheme based on component beams based on a simplified processing algorithm. Simulation results show that SIR of users in beamedge increases by $6 \mathrm{~dB}$ when using the joint transceiving scheme.
\end{abstract}

Keywords - Satellite, Multi-beam, beamforming, Least Mean Square, joint transceiving.

\section{INTRODUCTION}

Nowadays, multi-beam technology are widely used in satellite systems. Multi-beam satellite systems employ multiple beams to cover the service area, and make every beam transmit its own signals. When arranging these beams as honeycomb, cellular network of terrestrial systems can be easily used in satellite systems.

Generally, crossover area of two adjacent beams is considered to have $3 \mathrm{~dB}$ power attenuation in [1]. So in satellite cellular network, when users are in the edge area of their service beam, they may suffer from decrease of useful signal that leads to poor SINR. This problem also exists in terrestrial cellular network. To solve this problem, terrestrial system employs joint transceiving that makes adjacent cells transmit signals together. For terrestrial joint transceiving, one of the key problems is how to share information among cells which are physically separated. As the number of cells may be huge, it's virtually impossible to share all information. But for satellite systems, all information is transmitted via satellite, so there's no need to concern about sharing information among beams. Hence, joint transceiving seems to be an ideal method for satellite systems.

Some joint transceiving methods adopt Multiple Input Multiple Output (MIMO) space-time coding in [2]. But for satellite systems, channels are usually correlated Rayleigh fading channels with strong Line Of Sight (LOS) component Ref. [3] has shown that MIMO space-time codes suffers from serious performance degradation in such channels. So another method is needed to adapt joint transceiving to satellite systems. One method of joint transceiving is to make adjacent beams transmit the same signal and achieve coherent gain in beam-edge area. Due to this method, we need to insure that the phase of signal equal at the location of users. However the traditional method which employs channel estimation to estimate phase of every beam to adjust phase of signals to achieve the same is complex may delay because of the long distance between satellite and users. In this paper, we solve this problem by using a method of joint transceiving by composiᄀting beams for satellite systems. Recently, many scholars have studied the DBF algorithm and used it in many fields like adaptive beamforming, smart antennas and multibeam satellites in [4]-[10]. In these researches, the least mean square algorithm is considered to be an ideal algorithm which can be extended and used for the purpose of this paper. In this paper we proposed a joint transceiving scheme based on multiple component beams in which a simplified processing algorithm is used.

The rest of this paper is organized as follows. Section II outlines system model. In Section III, a joint transceiving scheme for satellite systems is described. Then numerical calculation results are provided in Section IV. Finally, Section $\mathrm{V}$ concludes the paper.

\section{SySTEM MODEL}

In multi-beam satellite systems, multiple composite beams compose coverage area. These beams are arranged as honeycomb so that frequency reuse can be used in multi-beam satellite network as well. In the case of satellite system, positions of users in service beam are expressed by points in spherical coordinates whose origin point is located at satellite. For beam $m$, its pattern is expressed as: $E_{m}(\Phi, \Psi)$, where $\Phi$ and $\Psi$ are parameters in spherical coordinates $(R, \Phi, \Psi)$. As $R$ only effects path attenuation and phase changing, there's no need to put it as parameter in expression of patterns. Multibeam satellite cellular coverage is shown in Figure 1.

For traditional frequency reuse schemes, the whole spectrum is divided into $F R$ equal parts and each $F R$ adjacent beams form a cluster. $F R$ is also known as frequency reuse factor, and can be expressed as 


$$
F R=i^{2}+i j+j^{2}
$$

where $i$ and $j$ are nonnegative integers. For example, $F R$ can be $1,3,4,7$, and so on.

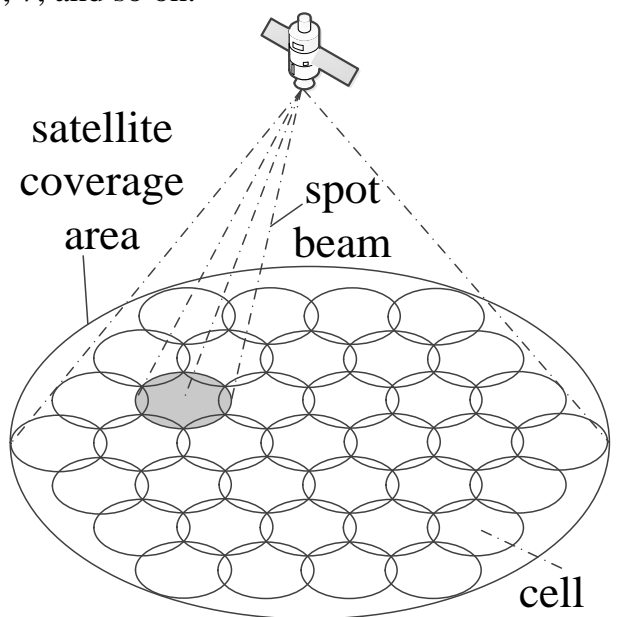

Fig. 1 Multi-beam satellite cellular coverage

If $F R$ is small, the system will get high spectral efficiency, but the inter-beam interference will be severe and limit the system capacity. If the system adopts a big $F R$, the inter-beam interference will be low but the system capacity is limited to the low spectral efficiency.

\section{III.Proposed JoInt TranSCEIVING SCHEME}

\section{A. Joint Transceiving Scheme for Satellite System}

The traditional joint transceiving method which was introduced in terrestrial mobile celluar networks need to estimate the channel real-time to obtain weighting coefficients, which requires additional processing and resources. In the environment of multi-beam GEO satellite system, the joint transceiving can also be used in which the channel estimation requirement may be relaxed for some extents.

As mentioned in Section I, users in beam-edge area suffer from useful signal power attenuation and need joint transceiving among adjacent beams. The sketch of joint transceiving is shown in Figure 2.

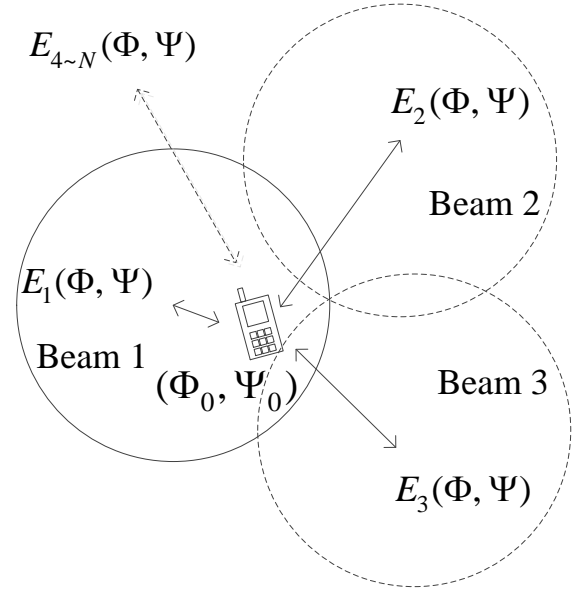

Fig. 2 Multi-beam Joint Transceiving Scheme
Figure 2 represents that beam 1 3 transmit the same signal to user served by beam 1 . The frequency reuse factor is larger than 3 which makes beam 2 and beam 3 not use the frequency of beam 1. So when joint transceiving is used, frequency resources of beam 2 and beam 3 do not reduce. Signals from other beams can be treated as interference, so SIR of user can be given by

$$
S I R=\left|\sum_{i=1}^{3} E_{i}(\Phi, \Psi) x_{i}\right|^{2} / \sum_{j=4}^{M}\left|a_{j} E_{j}(\Phi, \Psi) x_{j}\right|^{2},
$$

where $x_{i}$ is signal of user $i, a_{j}=1$ if beam $\mathrm{j}$ uses the frequency of user $i$ and $a_{j}=0$ if not.

When signals are equal-phase at location of user which means phases of $E_{i}(\Phi, \Psi) x_{i}$ are the same, SIR of user expressed will be maximized. That is main ideal of coherent joint transceiving.

\section{B. Joint Transceiver \& Weighted Factors}

A common joint transceiver is shown in Figure. 3.

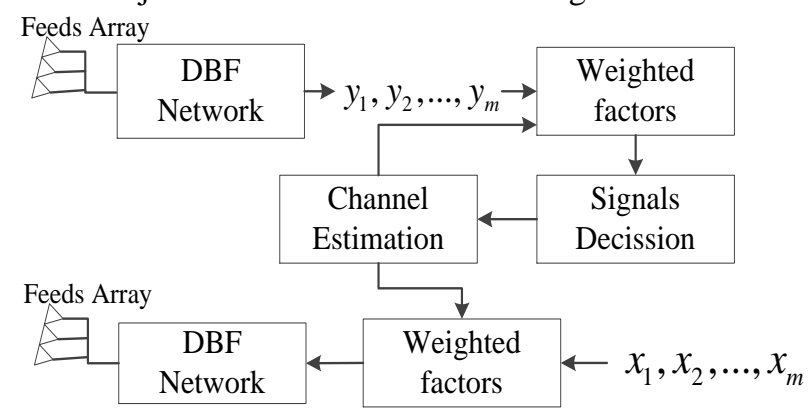

Fig.3 Common Joint Transceiver

$x_{1}, x_{2}, \ldots, x_{m}$ are signals transmitted by composite beams, and $y_{1}, y_{2}, \ldots, y_{m}$ are signals received. Generally channel estimation is needed at any time to generate weighted factors. However, as for joint transceiver, channel estimation is also necessary and weighted factors are constants during transmission and reception. Then the transceiver becomes an open-loop system, whose complexity is lower than the common one.

For downlink of multi-beam satellite systems, the received signal is characterized by

$$
y=\sum_{m=1}^{M} h_{m} x_{m}+n
$$

where $x_{m}$ is the signal transmitted by beam $m, h_{m}$ is the channel response from beam $m$ to receiver and $n \sim N(0,1)$ is i.i.d. proper Gaussian noise.

Here the channel response can be given by

$$
h_{m}=E_{m}(\Phi, \Psi)=A_{m}(\Phi, \Psi) e^{-j \theta_{m}(\Phi, \Psi)},
$$

where $A_{m}(\Phi, \Psi)$ is function of amplitude pattern and $\theta_{m}(\Phi, \Psi)$ is function of phase pattern.

When proceeding joint transceiving, several beams transmit the same signal. Without loss of generality, let beam 
1, 2 and 3 send the same signal. Then the received signal is given by

$$
y=\left(h_{1}+h_{2}+h_{3}\right) x_{0}+\sum_{m=4}^{M} h_{m} x_{m}+n,
$$

where $x_{0}$ is the useful signal. the SINR of received signal is:

$$
\operatorname{SINR}=\left|\left(h_{1}+h_{2}+h_{3}\right) x_{0}\right|^{2} /\left(\left|\sum_{m=4}^{M} h_{m} x_{m}\right|^{2}+n^{2}\right) .
$$

However, by compositing beams, channel response $h_{m}$ can be given by

$$
h_{m}=A_{m}(\Phi, \Psi) e^{-j\left[\theta_{m}+\delta_{m}(\Phi, \Psi)\right]},
$$

where $\delta_{i}(\Phi, \Psi)$ expresses the function of phase error and is far smaller than $\left|\theta_{m}\right|$.

As $\theta_{m}$ is known, weight factors can be given by $e^{j \theta_{m}}$. And employ approximate formula

$e^{-j \delta_{m}(\Phi, \Psi)} \approx 1-j \delta_{m}(\Phi, \Psi)$.

Then SINR is changed to

$$
\operatorname{SINR} \approx\left|\left(\left|h_{1}\right|+\left|h_{2}\right|+\left|h_{3}\right|-\delta\right) x_{0}\right|^{2} /\left(\left|\sum_{m=4}^{M} h_{m} x_{m}\right|^{2}+n^{2}\right),
$$

where $\delta=j\left[\delta_{1}(\Phi, \Psi)+\delta_{2}(\Phi, \Psi)+\delta_{3}(\Phi, \Psi)\right]$ is so small that SINR under these weighted factors is nearly maximized.

For uplink of multi-beam satellite systems, received signal of each beam is given by

$$
y_{m}=\sum_{n=1}^{N} h_{n m} x_{n}+n_{m}
$$

where $x_{n}$ is the signal transmitted by user $n, h_{n m}$ is the channel response from user $n$ to beam $m$ and $\sim N(0,1)$ is i.i.d. proper Gaussian noise.

Considering the same situation, let beam 1, 2 and 3 proceed joint reception. Adding the received signals of three beams, the sum is given by

$$
y=\sum_{m=1}^{3} \sum_{n=1}^{N} h_{n m} x_{n}+n,
$$

where $n$ is also i.i.d. proper Gaussian noise.

Supposing $x_{1}$ is the useful signal, and then SINR of $x_{1}$ is given by

$$
\operatorname{SINR}=\left|\left(h_{11}+h_{12}+h_{13}\right) x_{1}\right|^{2} /\left(\left|\sum_{m=1}^{3} \sum_{n=2}^{N} h_{n m} x_{n}\right|^{2}+|n|^{2}\right) .
$$

To maximize SINR, weighted factors $h_{1 m}{ }^{*} /\left|h_{1 m}\right|$ are needed for signal addition. Then the received signal is given by $y=\sum_{m=1}^{3} h_{n m}{ }^{*} /\left|h_{n m}\right| \sum_{n=1}^{N} h_{n m} x_{n}+n$.

The maximized SINR is

$$
\operatorname{SINR}=\left|\left(\left|h_{11}\right|+\left|h_{12}\right|+\left|h_{13}\right|\right) x_{1}\right|^{2} /\left(\left|\sum_{m=1}^{3} e^{j \theta_{1 m}} \sum_{n=2}^{N} h_{n m} x_{n}\right|^{2}+|n|^{2}\right)
$$

where $\theta_{1 m}$ is the phase of $h_{1 m}$.
Similar as downlink, by compositing beams, $h_{n m}$ can be given by

$$
h_{n m}=A_{m}\left(\Phi_{n}, \Psi_{n}\right) e^{-j\left[\theta_{m}+\delta_{m}\left(\Phi_{n}, \Psi_{n}\right)\right]} .
$$

Weighted factor as $e^{j \theta_{m}}$ is used here as well. Then SINR of $x_{1}$ is given by

SINR $=$

$$
\left|\left(\left|h_{11}\right|+\left|h_{12}\right|+\left|h_{13}\right|-\delta\right) x_{1}\right|^{2} /\left(\left|\sum_{m=1}^{3} e^{j \theta_{m}} \sum_{n=2}^{N} h_{n m} x_{n}\right|^{2}+|n|^{2}\right)^{\prime}
$$

where $\delta=j\left[\delta_{1}\left(\Phi_{1}, \Psi_{1}\right)+\delta_{2}\left(\Phi_{1}, \Psi_{1}\right)+\delta_{3}\left(\Phi_{1}, \Psi_{1}\right)\right]$.

As discussed in downlink, SINR expressed here is also nearly maximized.

\section{A Simplified Processing Algorithm}

As the propagation paths of adjacent beams is approximately equal to the same user, the users in beam-edge can sum and receive signal easily. Hence, we can simplify the received signal based on the equal-gain combination algorithm.

The equal-gain combination algorithm is not any sense of the best way to merge. Only assume that every signal under the same condition of SNR all the way, it is the best result on the meaning of the maximum SNR. Its output is the result of the multiple signals amplitude superposition. It is used to simplify the receiving signals in many systems. In equal-gain combination algorithm, all the received signals are summed coherently.

Generally, $\theta_{m}(\Phi, \Psi)$ is not equal so that the sum of $h_{m}$ cannot be maximized. To solve this problem, useful signals transmitted by different beams should be weighted with factors as $h_{m}{ }^{*} /\left|h_{m}\right|$. So SINR of received signal is changed to

$$
\begin{aligned}
\operatorname{SINR} & =\left|\sum_{m=1}^{3}\left(h_{m} x_{0} h_{m}{ }^{*} /\left|h_{m}\right|\right)\right|^{2} /\left(\left|\sum_{m=4}^{M} h_{m} x_{m}\right|^{2}+n^{2}\right) \\
& =\left|\left(\left|h_{1}\right|+\left|h_{2}\right|+\left|h_{3}\right|\right) x_{0}\right|^{2} /\left(\left|\sum_{m=4}^{M} h_{m} x_{m}\right|^{2}+n^{2}\right)
\end{aligned}
$$

Since $h_{1}+h_{2}+h_{3} \leq\left|h_{1}\right|+\left|h_{2}\right|+\left|h_{3}\right|$, weighted factors $h_{m}{ }^{*} /\left|h_{m}\right|$ is optimal. As the weighted factors contain parameter of channel response $h_{m}$, channel estimation is needed at any time.

\section{IV.Simulation RESULTS}

In this section, the performance of the joint transceiving scheme is simulated.

The antenna parameters are described in Table 1.

TABLE 1. ANTENNA PARAMETERS

\begin{tabular}{|l|l|}
\hline Parameter & Value \\
\hline Wavelength $(\lambda)$ & $15 \mathrm{~cm}$ \\
\hline Focal length $(f)$ & $7.443 \mathrm{~m}$ \\
\hline Diameter $(D)$ & $12.5 \mathrm{~m}$ \\
\hline Offset height $(H)$ & $2.607 \mathrm{~m}$ \\
\hline
\end{tabular}


In this simulation, the max phase error is small. This error may bring negative effect on using low complexity joint transceiving. In extreme case, 3 beams have 0.32 radian phase difference to each other. Then it can be calculated that the joint power gain is $6.23 \mathrm{~dB}$. Comparing with the ideal condition that the joint power gain is $6.53 \mathrm{~dB}$, the phase error results in $0.3 \mathrm{~dB}$ deterioration. However, such deterioration is so small that performance can also get promoted in this extreme case. Therefore, least mean square method with multiple reference points is proved useful in forming beams.

Figure. 4 shows users' SIR in the central beam from beam center to beam edge.

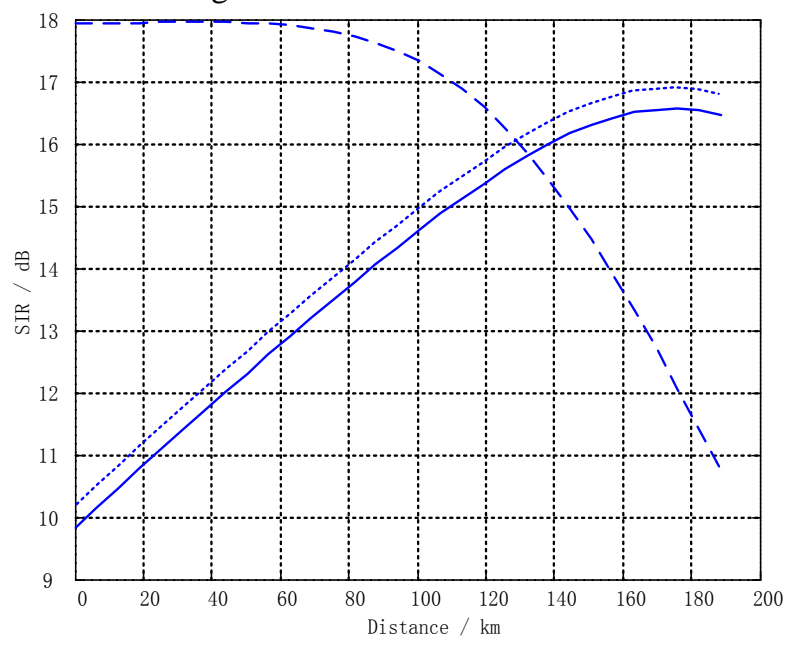

Fig.4 SIR of users in central beam

The horizontal axis shows the distance from beam center to user. Here the satellite is considered to be located in GEO, so radius of beam is about $200 \mathrm{~km}$. Solid curve shows the user using 3-beam coherent joint transceiving, sparse dashed curve shows the user not using joint transceiving and dense dashed curve shows the user using joint transceiving in ideal situation. Frequency reuse factor here is 3 , and interference caused by coherent joint transceiving is also taken into consideration.

It can be seen from Figure 3 that when user is more than $130 \mathrm{~km}$ away from beam center, 3-beam coherent joint transceiving can achieve better performance. When users are in crossover of beams, SIR improvement can attain $6 \mathrm{~dB}$. Compared with ideal situation, SIR of case that using low complexity joint transceiving is about $0.3 \mathrm{~dB}$ less. This disadvantage is small relative to the advantage.

Furthermore, SIR improvement is lower than signal power gain which is considered to be above $6.23 \mathrm{~dB}$. The reason is that when using coherent joint transceiving, some beams will break the frequency reuse, then the interference will be greater and make SIR lower. Fortunately, this interference seems to be small and improvement of coherent joint transceiving is still prominent. For actual systems which is going to use coherent joint transceiving, a new resource allocation scheme may be needed to reduce the interference mentioned above.

\section{Conclusions}

In this paper, we propose a joint transceiving scheme for multi-beam satellite communication systems. First, a method is modified to meet the requirement of compositing beams. Then, the low complexity joint transceiving under composite beams based on a simplified processing algorithm is presented. Lastly, simulation result shows that beams are successfully composited and users' SIR in beam-edge achieve $6 \mathrm{~dB}$ improvement by using our low complexity joint transceiving scheme.

\section{ACKNOWLEDGMENT}

This work is partly supported by the National Science Foundation of China (Grant No. NFSC \#61071083, \#61371073) and the National High-Tech Research and Development Program of China (863 Program), No.2012AA01A506. Corresponding author: Jianjun Wu, Email: just@pku.edu.cn.

\section{REFERENCES}

[1] MONTGOMERY, J. P., D. L. RUNYON, and J. A. FULLER. "Large multibeam lens antennas for EHF SATCOM." Military Communications Conference, 1988. MILCOM 88, Conference record. 21st Century Military Communications-What's Possible? 1988 IEEE. IEEE, 1988.

[2] GESBERT, DAVID, et al. "Multi-cell MIMO cooperative networks: A new look at interference." Selected Areas in Communications, IEEE Journal on 28.9 (2010): 1380-1408.

[3] H. BOLCSKEI and AROGYASWAMI J. PAULRAJ. "Performance analysis of space-time codes in correlated Rayleigh fading environments." Proc. Asilomar Conf. on Signals, Systems and Computers, Pacific Grove, CA, Nov. 2000, vol.1, pp. 687-693.

[4] WIDROW, BERNARD, et al. "Adaptive antenna systems." Proceedings of the IEEE55.12 (1967): 2143-2159.

[5] FEUER, ARIE, and EHUD WEINSTEIN. "Convergence analysis of LMS filters with uncorrelated Gaussian data." Acoustics, Speech and Signal Processing, IEEE Transactions on 33.1 (1985): 222-230.

[6] CHOI, SEUNGWON, and DONGHEE SHIM. "A novel adaptive beamforming algorithm for a smart antenna system in a CDMA mobile communication environment." Vehicular Technology, IEEE Transactions on 49.5 (2000): 1793-1806.

[7] LI WINSTON, XIPING HUANG, and HENRY LEUNG "Performance evaluation of digital beamforming strategies for satellite communications." Aerospace and Electronic Systems, IEEE Transactions on 40.1 (2004): 12-26.

[8] KLEIN, C. "Design of shaped-beam antennas through minimax gain optimization." Antennas and Propagation, IEEE Transactions on 32.9 (1984): 963-968.

[9] CHU, TA-SHING, and R. TURRIN. "Depolarization properties of offset reflector antennas." Antennas and Propagation, IEEE Transactions on 21.3 (1973): 339-345.

[10] RAHMAT-SAMII, Y. A. H. Y. A., and SHUNG-WU LEE. "Directivity of planar array feeds for satellite reflector applications." Antennas and Propagation, IEEE Transactions on 31.3 (1983): 463-470.

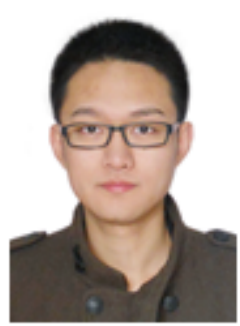

Gao Yazhe, is an undergraduate student in ICES, Beijing Information S\&T University, Beijing, P.R. China. His research interests are in the area of satellite mobile communications and wireless communications. Email: yazhegao@163.com. 
Wu Jianjun, received his B.S., M.S. and Ph.D. degree from Peking University, Beijing, P. R. China, in 1989, 1992 and 2006, respectively. Since 1992, he has joined the School of Electronics Engineering and Computer Science, Peking University, and has been appointed as an associate professor since 2002. His research interests are in the areas of satellite communications, wireless communications, and communications signal processing. *The corresponding author. Email: just@pku.edu.cn. 